\title{
Lavender oil supplementation for the management of anxiety disorder
}

\author{
Henneman $\mathrm{A}^{1 *}$, Axtell $\mathrm{S}^{2}$, Chaudry $\mathrm{S}^{3}$ and Younas $\mathrm{W}^{3}$ \\ ${ }^{1}$ Associate Professor of Pharmacy Practice, Palm Beach Atlantic University, West Palm Beach, Florida, USA \\ ${ }^{2}$ Assistant Professor of Pharmacy Practice, Palm Beach Atlantic University, West Palm Beach, Florida, USA \\ ${ }^{3}$ Pharm.D. Candidate, Palm Beach Atlantic University, West Palm Beach, Florida, USA
}

\begin{abstract}
Purpose: Available evidence for the use of lavender oil as a means of managing anxiety disorders, such as generalized anxiety disorder is reviewed.

Summary: Many patients seek alternative or complementary therapies as a means to manage various disease states in lieu of or in addition to traditional treatment modalities. Lavender essential oil is one such complementary therapy available in a variety of dosage forms with many reported uses including management of anxiety and anxiety disorders. A Medline search was conducted for clinical trials or case reports of lavender oil for the management of anxiety disorder. Five clinical studies were identified, all examining the use of an oral lavender oil dosage form. Overall, the majority of patients experienced an improvement in anxiety symptoms based on change in HAM-A score with few, mild side effects.
\end{abstract}

Conclusion: The use of an oral lavender oil dosage form appears to improve anxiety symptoms in patients with chronic anxiety such as generalized anxiety disorder with minimal side effects, however evidence is limited.

\section{Introduction}

With an estimated $38 \%$ of adult Americans utilizing some form of complementary health approach and $\$ 12.8$ billion being spent on natural supplements in the United States, the need for pharmacists to be able to provide education regarding proper use of supplements remains high [1]. One such type of complementary medicine involves the use of essential oils, which are marketed as alternative therapy to manage a wide range of health conditions. Essential oils are volatile organic plant constituents often used as part of a holistic approach to managing disease and promoting well-being [2]. The oils may be absorbed through inhalation via the respiratory system, transdermally via direct contact, or oral ingestion [3].

One such health condition essential oils, specifically lavender oil, have been used to help manage are chronic anxiety disorders, most commonly classified as generalized anxiety disorder (GAD). GAD is estimated to affect approximately 6.8 million adults in the United States [4]. It can be defined as ongoing excessive worry and anxiety that can result in severe distress and impairment. Generalized anxiety disorder is often chronic and persistent in nature and has been associated with poor health outcomes. It often requires pharmacological treatment combined with cognitive behavioral therapy for management. The current first line drug options include selective serotonin reuptake inhibitors (SSRIs), serotonin norepinephrine reuptake inhibitors (SNRIs), and pregabalin. Second line agents include imipramine, buspirone and benzodiazepines [5]. Many of these drugs may cause adverse effects as well as safety concerns, particularly chronic use of benzodiazepines; therefore, alternative methods for managing GAD while maintaining safety are of particular interest [6].

Lavender essences have been recognized for their calming and sedative properties dating back centuries $[7,8]$. Several small studies have been conducted evaluating the effects of lavender oils on various types of both acute and chronic anxiety [9-17]. The active substance is typically produced through steam distillation of Lavandula angustifolia flowers, or English lavender, though other species may be used [8]. The production of active compounds found within the lavender plant varies depending on the species and the manner in which the compounds are isolated. This production process has the potential to produce variation amongst marketed essential oil products. Cultivation of lavender hybrid species is common to increase essential oil yields [3].

The use of lavender oil as an anxiolytic has been demonstrated in various clinical trials for anxiety, sleep and mood disorders, as well as posttraumatic stress disorder. Furthermore, it has been approved in Germany for treatment of restlessness related to anxiety [18]. The product is available commercially in Germany as Silexan $80 \mathrm{mg}$ softgel capsules, with a recommended dose of 1 capsule by mouth daily [19]. Though no commercially FDA approved products are available in the United States, lavender is among the FDA's list of substances that are generally recognized as safe (GRAS) for consumption (FDA) [20]. The mechanism of action is not completely understood but has been proposed to modulate gamma-aminobutyric acid (GABA) similar to benzodiazepines and possibly cause serotonin modulation, similar to SSRI/SNRIs [21]. Given the benefits Silexan has shown in sleep, mood and acute anxiety, its potential use for generalized anxiety disorder

${ }^{\star}$ Correspondence to: Henneman A, Pharm.D., BCPS, BCACP, CDE, Associate Professor of Pharmacy Practice, Palm Beach Atlantic University, West Palm Beach, Florida, USA, Tel: (561) 803-2721; E-mail: Amy_Henneman@pba.edu

Key words: lavender oil, anxiety, essential oils, generalized anxiety disorder

Received: March 25, 2018; Accepted: June 06, 2018; Published: June 08, 2018 
should be further investigated. This article will analyze and evaluate the data supporting the use of lavender essential oil for the management of chronic anxiety and GAD.

\section{Literature review}

A computerized search of the published literature was conducted using Medline to locate clinical trials and or case reports pertaining to the use of lavender essential oils in the management of chronic anxiety and GAD. The search was limited to English-language journals and human studies. There was no limitation set on the year of publication. Keywords of the search included lavender, essential oil, generalized anxiety disorder, chronic anxiety disorder, and anxiety disorder. Additionally, any relevant articles referenced in articles retrieved from the Medline search were utilized. Standard tertiary drug resources were utilized for preliminary knowledge of lavender's safety, pharmacokinetics, and pharmaceutical information. The use of lavender essential oil in the management of chronic anxiety, primarily GAD, was noted in five clinical studies.

\section{Evidence of Interaction}

Woelk, et.al. [13] examined the efficacy of lavender oil in the German approved formulation of Silexan versus lorazepam in a 6 week randomized, parallel, multi-center, double-blind study in Germany. Seventy-seven patients were randomized to either the Silexan group $(n=40)$ or the lorazepam group $(n=36)$. The Silexan group received one $80 \mathrm{mg}$ lavender capsule and one lorazepam placebo capsule while the lorazepam group received one $0.5 \mathrm{mg}$ lorazepam capsule and one lavender placebo capsule. Patients were included in the study if they were between the ages of 18-65 years old with a primary diagnosis of GAD based on Diagnostic and Statistical Manual of Mental DisordersIV (DSM-IV) and current outpatient treatment. Patients also needed to have selected Hamilton Anxiety Rating Scale (HAM-A) Item 1 "anxious mood" $\geq 2$ and Item $\geq 2$ "tension" $\geq 2$ as well as have a total HAM-A score of $\geq 18$. A wash-out period of one week was required prior to study entry and patients with a decrease of $\geq 25 \%$ in their HAM-A total score during this time were excluded. Neither power nor the needed noninferiority margin were noted for the study.

The primary outcome was the change in HAM-A total score from baseline to week 6. Secondary outcomes included a comparison of responder and remission rates between Silexan and lorazepam as well as change in the Zung's Self-rating Anxiety Scale (SAS), the Clinical Global Impression (CGI), Penn State Worry Questionnaire past week version (PSWQ-PW), the SF-36 Health Survey Questionnaire and patient's Sleep Diary. Safety was assessed through physical exams, vital signs, ECG and laboratory monitoring.

The HAM-A total score was $25+4$ points in both treatment arms at baseline. By week 6 , it decreased to $11.3+6.7$ points in the Silexan group and $11.6+6.6$ points in the lorazepam group. The values were similar for the intention-to-treat (ITT) and per protocol (PP) data sets. The authors noted Silexan to be noninferior to lorazepam $(\mathrm{p}=0.05)$. Forty percent of responders in the Silexan group $(n=16)$ and $27 \%$ of responders in the lorazepam group $(n=10)$ achieved remission $(\mathrm{p}=0.04)$. Similar improvements between both groups were noted in the secondary outcomes. No serious adverse events were noted in either group during the study. The majority of adverse events in the Silexan group were gastrointestinal related and included nausea, belching, breath odor, and dyspepsia. Of those noted in the lorazepam group, fatigue was the most common.
The authors concluded that Silexan is not less effective than lorazepam $0.5 \mathrm{mg}$ capsule in the treatment of patients with GAD, however several limitations exist. Baseline characteristics were not noted with the exception of the majority of the patients being female (76.6\%). Information regarding comorbid conditions or concomitant medications was not provided. Though there was a washout period, the types of medications that were discontinued are also not noted. The short duration of this study makes it difficult to know if the effects of lavender oil in patients with generalized anxiety would persist long term. Lastly, while the authors stated inferiority could be excluded based on a HAM-A difference between the 2 groups of 2.8, the needed noninferiority margin as well as power were not provided.

Kasper, et al. [14] examined lavender oil's efficacy in reducing anxiety versus placebo in a prospective, randomized, double-blind, multi-center study that took place in 27 general or psychiatric primary care centers in Germany. Two hundred sixteen patients with anxiety disorder according to the DSM-IV or the International Statistical Classification of Diseases and related Health Problems, $10^{\text {th }}$ edition (ICD-10) were randomized to either Silexan $80 \mathrm{mg}$ daily $(\mathrm{n}=107)$ or placebo $(n=109)$. Additionally, participants had to have a HAM-A total score $\geq 18$ points and $\geq 2$ points for items anxious mood and insomnia, as well as $\geq 5$ points on the Pittsburgh Sleep quality Index (PSQI). Patients were excluded if they also had any clinically important psychiatric or neurological diagnosis other than anxiety disorder, risk of suicide, or substance abuse disorder as well as a decrease $\geq 25 \%$ on the HAM-A during the run-in phase. Other psychotropic medication or muscle relaxant use were excluded. The primary outcome was the change in the HAM-A and PSQI from baseline to week 10. Secondary outcomes included the CGI, observer rating scale, SAS, and the SF-36 Health Survey Questionnaire. Two hundred twenty participants were needed to detect a difference of $2.5+6.0$ points on the HAM-A and a difference of $1.0+2.7$ points on the PSQI and achieve $80 \%$ power.

Baseline characteristics were similar between groups. The majority of participants were female. The average HAM-A score was approximately 27 and the PSQI score was 12. For the primary outcome, the HAM-A score decreased $16.0+8.3$ points for the Silexan group and $9.5+9.1$ points for the placebo group $(\mathrm{p}<0.001)$ by week 10 . The PSQI score decreased $5.5+4.4$ points for the Silexan group and $3.8+4.1$ points for the placebo group $(\mathrm{p}=0.002)$. The reported rate of adverse events was $36.4 \%$ for the Silexan group and $32.1 \%$ for the placebo group. Of those deemed potentially attributable to the study medication, the most common were gastrointestinal related with dyspepsia and eructation. The authors noted Silexan to be superior to placebo in this study.

The small sample size and short duration of this study limit extrapolation. Also, similar to other studies the patient population was mostly female. Other psychiatric or neurological diseases were excluded, however the types of comorbid diseases participants may have had were not noted. The washout period, though brief, may have limited patients willing to participate since they would have to go without medication for 3 to 7 days, however placebo appears to have been given during this time. Lastly, the study possessed two primary outcomes which may increase the chance of a Type 1 error impacting the significance of the study results.

Kasper, et al. [15] examined lavender oil's efficacy in reducing anxiety in a prospective, randomized, double-blind, double-dummy, placebo-controlled, multi-dose, per-protocol, multi-center trial. Five hundred and thirty-nine adult patients (ages $18-65$ ) with GAD who scored $\geq 18$ points on HAM-A and selected $\geq 2$ items for "anxious mood" and "tension". Additionally, a subscore of $<21$ was needed for 
psychic anxiety and a Covi Anxiety Scale (CAS) score $\geq 9$. Patients were recruited from 57 general and psychiatric practices in Germany. Additionally, patients had to score $\geq 2$ points for HAM-A items of anxious mood and tension, a score of at least 21 for psychic anxiety, and a Covi Anxiety Scale score of $\geq 9$. Exclusion criteria included patients taking psychotropic drugs or undergoing psychotherapy, patients with another DSM-IV-TR Axis I diagnosis within 6 months of study entry, predominant or severe depressive symptoms, risk of suicide, substance abuse, and schizophrenia. This study employed the use of a treatment free screening and a washout period of 3 to 7 days. Patients were then selected and randomized into either Silexan $160 \mathrm{mg}(\mathrm{n}=128)$, Silexan $80 \mathrm{mg}(\mathrm{n}=135)$, paroxetine $20 \mathrm{mg}(\mathrm{n}=137)$, or placebo $(\mathrm{n}=136)$ daily. Safety and efficacy were evaluated every 2 weeks for a total of 10 weeks. The primary outcome was a measured reduction in the HAM-A total score from baseline to the end of treatment. Secondary outcomes included response and remission assessed via HAM-A total scores, CAS, Hamilton Rating Scale for Depression (HAMD) and the CGI. Safety was measured by patient reported adverse events, physical exams, and routine lab measurements. One hundred thirty patients in each treatment arm were needed to attain a power of $80 \%$ and detect a three point difference between Silexan and placebo for change in HAM-A total score versus baseline. An interim analysis was conducted and the one-sided a priori alpha was set at 0.0152 . The trial was not powered to assess the change in HAM-A score for paroxetine versus Silexan or placebo.

Baseline characteristics were similar across all treatment arms, except patients in the Silexan $160 \mathrm{mg}$ group had higher overall anxiety scores versus placebo ( $\mathrm{p}<0.05$ ). Overall, $75 \%$ of the patients were female and all but 2 Caucasian. Most of the patients had been suffering from GAD for about 2.5 years in all treatment groups. Silexan $160 \mathrm{mg} / \mathrm{d}$ was shown to be superior to placebo after 4 weeks of treatment with a mean difference to placebo of 2.6 points $(p<0.001)$. After 6 weeks of treatment, a statistically significant difference was noted between Silexan $80 \mathrm{mg} / \mathrm{d}$ and placebo, with a mean difference of 2.3 points $(p=0.02)$. The effect remained statistically significant until the end of treatment. While a decrease in the HAM-A score was noted with paroxetine versus placebo, statistical significance was not achieved at any measured point throughout the duration of the student. Similar treatment group differences were also noted across all secondary endpoints. The reported rate of adverse events was $25 \%$ for the Silexan $160 \mathrm{mg} /$ day group, $34.8 \%$ for the Silexan $80 \mathrm{mg} /$ day group, $40.9 \%$ in the paroxetine group, and $30.9 \%$ in the placebo group. The authors noted the most common adverse events were gastrointestinal, infection, and nervous system disorders, however the specific event was not given. The authors of this study concluded Silexan at both $160 \mathrm{mg} / \mathrm{d}$ and $80 \mathrm{mg} / \mathrm{d}$ was efficacious in reducing the symptoms of anxiety in patients with GAD over a 10 week period.

The majority of the participants in this study were Caucasian women, limiting extrapolation to other populations. Patients taking other psychotropic medications were excluded from the study, however the authors provide no information regarding what medications they considered to be classified as a psychotropic. Additionally, while the authors did include a washout period of 3-7 days, depending on the medication the patient was discontinuing, this may not have permitted adequate time for the medications' effects to subside. Lastly, the short duration of the study makes it difficult to determine if the differences seen between treatment arms would be noted long term.

Kasper, et al. [16] conducted a second study in Germany which was a 10 -week, double-blind, multicenter, randomized trial comparing the efficacy of Silexan $80 \mathrm{mg}$ daily to a matching placebo in patients suffering from anxiety with dominant symptoms of disturbed sleep and restlessness. Participants were included if they were between the ages of 18-65 years old and suffered from agitation and restlessness (per ICD criteria R45.1), while scoring $\geq 18$ on the HAM-A. Additionally, they needed a minimum of two points each in the sections of "tension" and "insomnia", as well as a score of $\geq 6$ on the PSQI indicating disturbed sleep. Individuals with either psychiatric or neurological disorders, mild/moderate depression, personality disorders, insomnia, suicidal ideation, history of substance abuse, undergoing psychotherapy, or use of either psychotropic medications or muscle relaxants were excluded from this trial. The study's co-primary outcomes were the absolute change in HAM-A score and PSQI score. Secondary outcomes included HAM-A sub scores, CGI score, SAS, and the State Check (SC) scale. One hundred fifty-six patients were needed to detect a 3 point mean value difference in the HAM-A scale and a 1.5 point mean value difference in the PSQI. Power was set at $80 \%$.

One hundred and seventy patients were randomized to receive either Silexan $(n=86)$ or placebo $(n=84)$. Baseline demographics were similar between treatment arms and primarily consisted of Caucasian females considered "markedly or severely ill." The total HAM-A score in the Silexan group decreased from an average of $25.5+6.0$ points to $13.7+7.0$ points. The placebo group decreased from $26.5+6.1$ points to $16.9+9.8$ points. From week 4 to week 10 , a statistically significant difference was found between the two groups $(\mathrm{p}<0.05)$. Results regarding treatment effect on PSQI scores were not given. Authors stated superiority of Silexan versus placebo could not be determined for either HAM-A or PSQI based mostly on the small change noted for the PSQI total score $(\mathrm{p}=0.091)$. Statistical significance was not met for the primary outcome as a decrease in both the HAM-A and PQSI scores was required. Reported adverse events with Silexan use occurred in $10.5 \%$ of participants and included eructation, diarrhea, oral discomfort, and gastritis. No serious adverse events were seen with Silexan use. Although statistical significance was not achieved with the co-primary outcomes, the authors concluded Silexan showed anxiolytic impact, but did not improve patients sleep.

Limitations of this study included the large percentage of Caucasian females, limiting extrapolation to other populations. The authors did note the use of a self-developed scale to assess restlessness which has not been validated as of yet. In addition, information regarding participants' comorbidities, lifestyle changes or events during the study period (exercise, smoking, tragedy, etc.), and concomitant medications (in addition to psychotropic medications and muscle relaxants) were not noted. This information would have been helpful in determining if confounders were present. Similar to other studies, this study was relatively small and of short duration making it difficult to determine long-term effects of Silexan treatment.

In another study, Kasper, et a., [17] compared the efficacy, safety, and tolerability of Silexan $80 \mathrm{mg}(\mathrm{n}=159)$ daily to placebo $(\mathrm{n}=156)$ over a 10-week period throughout multiple centers in Germany. This double-blind, randomized, parallel, multicenter trial included participants aged 18-65 years who had a diagnosis of Mixed Anxiety and Depressive Disorder (MADD) according to ICD-10 category F41.2. Participants had to possess a HAM-A score of $\geq 18$ AND score $\geq 2$ points for both HAM-A items of "anxious mood" and "depressed mood." Patients who reported a history of suicidal attempts or "autoaggressive" behavior, possessed $\geq 2$ points under the "suicidal thoughts" item within the HAM-A score, possessed neurological or psychiatric diagnoses other than MADD, reported a history of substance abuse, or 
were previously (within 30 days of randomization) or concomitantly undergoing psychotherapy or utilizing psychotropic medications, were excluded from the study.

The study's two primary outcomes were the change in participants' HAM-A score and Montgomery Asberg Depression Rating Scale (MADRS) score. Secondary outcomes included total score (HAM-A and MADRS) reduction of $\geq 50 \%$ and a total score of $<10$ points on the HAM-A AND $<10$ points for MADRS, as these were considered remission, as well as State-trait Anxiety Inventory (STAI), self-rating of anxiety, the self-rated Hospital Anxiety and Depression Scale (HADS), the CGI scale, the Sheehan Disability Scale (SDS), and the SF-36 health survey questionnaire. A sample size of 157 patients in each treatment group was needed to detect a mean value difference of 3 points and provide $90 \%$ power for the first primary outcome and $81 \%$ power for the second primary outcome. One sided alpha error was set a priori at 0.0152 . Participants' baseline demographics were similar between treatment arms and consisted predominantly of Caucasian women. Almost $40 \%$ of participants were either current smokers or ex-smokers and approximately $7 \%$ drank alcohol more than twice weekly. The study states participants' length of MADD diagnosis, comorbidities, alcohol use, concomitant medication use, and smoking statuses were similar between treatment arms, but no p-values were provided.

The HAM-A total score decreased $10.8+9.6$ points for the Silexan group and $8.4+8.9$ points for the placebo group at 10 weeks $(\mathrm{p}=0.02)$. The MADRS total score decreased $9.2+9.9$ points for the Silexan group and $6.1+7.6$ points for the placebo group at 10 weeks $(p<0.01)$. Per the authors, a statistically significant difference was noted from week 4 until the end of the study for change in both the HAM-A and MADRS total score. Adverse effects deemed to be potentially related to the study medication included eructation ( $10 \%$ vs $0 \%)$, headache $(2.5 \%$ vs $5.7 \%)$, diarrhea $(1.3 \%$ vs $2.5 \%)$, and nausea $(1.9 \% 0.6 \%)$. The authors concluded that Silexan showed not only an anxiolytic effect, but an antidepressant effect, making it efficacious in patients suffering from MADD.

Limitations include confounding factors such as tobacco/alcohol use, exercise, and/or other lifestyle modifications or events that may impact participants' anxiety levels were not noted. Additionally, psychotropic medication use was not permitted during the study, but further mention of other medications and/or comorbid conditions was not noted. Lastly, this study also employed two primary outcomes, allowing the chance of a Type 1 error to increase.

\section{Discussion}

Based on the above studies, lavender essential oil may have a role in the management of chronic anxiety disorders such as GAD. One of the strengths of the studies is that each used the same lavender oil formulation, Silexan, which is an approved formulation in Germany. However, since this formulation is not approved for use in the United States, it is difficult to know if different formulations would produce similar effects. Additionally, the supplement was given orally in all studies. Lavender essential oils are available in various dosage forms which may not produce the same effects as those noted in the reviewed studies. No studies of lavender in other dosage forms for the treatment of anxiety were identified. All studies utilized a HAM-A score of at least 18 as inclusion criteria for participation. Additionally, change in the HAM-A from baseline was a primary outcome of each study, and each study noted an improvement in the HAM-A score (Table 1). Improvements in anxiety based on changes in the HAM-A may be comparable to improvements seen with SSRIs and benzodiazepines. However, a direct comparison was not the primary endpoint in all
Table 1. Summary for the primary outcome

\begin{tabular}{|c|c|c|c|}
\hline Reference & Study duration & $\begin{array}{c}\text { Number of } \\
\text { participants }\end{array}$ & $\begin{array}{c}\text { Change in HAMA score with } \\
\text { oral lavender essential oil } \\
\text { (points) }\end{array}$ \\
\hline Woelk, et al. [13] & 6 weeks & 77 & $11.3 \pm 6.7$ \\
\hline Kasper, et al. [14] & 10 weeks & 216 & $16.0 \pm 8.3$ \\
\hline Kasper, et al. [15] & 10 weeks & 539 & $\begin{array}{c}(160 \mathrm{mg} / \mathrm{d}) 14.1 \pm 9.3 \\
(80 \mathrm{mg} / \mathrm{d}) 12.8 \pm 8.7\end{array}$ \\
\hline Kasper, et al. [16] & 10 weeks & 170 & $12.0 \pm 2.7$ \\
\hline Kasper, et al. $[17]$ & 10 weeks & 318 & $10.8 \pm 9.6$ \\
\hline
\end{tabular}

studies, and a limited number of SSRI and benzodiazepine medications were utilized in the comparator groups of these studies. Therefore, it is not known if lavender oil is as effective as SSRIs or benzodiazepines, particularly long term. Overall, lavender oil in a standardized capsule dosage form does appear to be more effective for the management of anxiety then placebo based on the evidence presented.

The reviewed studies did have several limitations. Patients with concomitant diagnosed mental disorders were excluded from each of these studies. However, within the general population, many patients suffer from more than one mental illness diagnosis [22]. Additionally, all studies had a brief washout period prior to study start. This requirement could have affected the types of patients who wished to participate in the study. Those with more severe anxiety may not have been willing to discontinue their current medication for even a short period of time. It should also be noted that none of the studies were longer than 10 weeks. Since anxiety disorders often persist over many years, the efficacy of oral lavender essential oil long term is unknown. Four of the 5 studies were conducted by the same investigators, so bias cannot be excluded.

Lavender oil when taken orally had few adverse effects with the most common being gastrointestinal related. However, potential adverse effects would be expected to vary dependent on the dosage form utilized. According to Natural Medicines Database, various other adverse effects have been noted when lavender essential oils have been utilized for various indications, including case reports of gynecomastia in young children utilizing a topical lavender oil formulation [23]. According to the review by Kasper et.al., ${ }^{19}$ lavender oil in the gel capsule dosage form (Silexan) does not appear to affect the cytochrome enzyme system nor plasma levels of an oral contraceptive containing ethinyl estradiol and levonorgestrel. None of the studies reviewed examined potential drug interactions with lavender oil, though medications for the treatment of various chronic disease states were allowed.

\section{Conclusions}

Based on available evidence, oral lavender essential oil may be effective in the management of chronic anxiety. However, since a standard dosage form is not approved for use in the United States, it is unknown if other dosage forms would produce similar results.

\section{Disclosures}

The authors have no financial support or personal connections that could be perceived to bias the manuscript, or any other potential conflicts of interest to disclose.

\section{References}

1. Nahin RL, Barnes PM, Stussman BJ (2016) Expenditures on Complementary Health Approaches: United States, 2012. Natl Health Stat Report 95: 1-11. [Crossref]

2. Louis M, Kowalski SD (2002) Use of aromatherapy with hospice patients to decrease pain, anxiety, and depression and to promote an increased sense of well-being. $\mathrm{Am} \mathrm{J}$ Hosp Palliat Care 19: 381-386. [Crossref] 
3. Woronuk G, Demissie Z, Rheault M, Mahmoud S (2011) Biosynthesis and therapeutic properties of Lavandula essential oil constituents. Planta Med 77: 7-15. [Crossref]

4. Kessler RC, Chiu WT, Demler O, Walters EE (2005) Prevalence, severity, and comorbidity of twelve-month DSM-IV disorders in the National Comorbidity Survey Replication (NCS-R). Arch Gen Psychiatry 62: 617-627. [Crossref]

5. Bandelow B, Zohar J, Hollander E, Kasper S, Möller HJ, et al. (2008) World Federation of Societies of Biological Psychiatry (WFSBP) guidelines for the pharmacological treatment of anxiety, obsessive-compulsive and post-traumatic stress disorders - first revision. World J Biol Psychiatry 9: 248-312. [Crossref]

6. Baldwin DS, Anderson IM, Nutt DJ, Bandelow B, Bond A, et al. (2005) Evidence-based guidelines for the pharmacological treatment of anxiety disorders: recommendations from the British Association for Psychopharmacology. J Psychopharmacology 19: 567596. [Crossref]

7. Cavanagh HM, Wilkinson JM (2002) Biological activities of lavender essential oil. Phytother Res 16: 301-308. [Crossref]

8. Denner SS (2009) Lavandula angustifolia Miller: English lavender. Holist Nurs Pract 23: 57-64. [Crossref]

9. Louis M, Kowalski SD (2002) Use of aromatherapy with hospice patients to decrease pain, anxiety, and depression and to promote an increased sense of well-being. Am J Hosp Palliat Care 19: 381-386. [Crossref]

10. Kritsidima M, Newton T, Asimakopoulou K (2010) The effects of lavender scent on dental patient anxiety levels: a cluster randomised-controlled trial. Community Dent Oral Epidemiol 38: 83-87. [Crossref]

11. Soden K, Vincent K, Craske S, Lucas C, Ashley S (2004) A randomized controlled trail of aromatherapy massage in a hospice setting. Palliat Med 18: 87-92. [Crossref]

12. Conrad P, Adams C (2012) The effects of clinical aromatherapy for anxiety and depression in the high risk postpartum woman - a pilot study. Complementary Therapies in Clinical Practice 18: 164-168. [Crossref]

13. Woelk H, Schläfke S (2010) A multi-center, double-blind, randomised study of the Lavender oil preparation Silexan in comparison to Lorazepam for generalized anxiety disorder. Phytomedicine 17: 94-99. [Crossref]
14. Kasper S, Gastpar M, Muller W (2010) Silexan, an orally administered Lavandula oil preparation, is effective in the treatment of subsyndromal anxiety disorder: a randomized, double-blind, placebo controlled trial. Int Clin Psychopharmacol 25: 277 287. [Crossref]

15. Kasper S, Gastpar M, Müller WE, Volz HP, Möller HJ, et al. (2014) Lavender oil preparation Silexan is effective in generalized anxiety disorder--a randomized, doubleblind comparison to placebo and paroxetine. Int J Neuropsychopharmacol 17: 859-869. [Crossref]

16. Kasper S, Anghelescu I, Dienel A (2015) Efficacy of orally administered Silexan in patients with anxiety-related restlessness and disturbed sleep - a randomized, placebocontrolled trial. Eur Neuropsychopharmacol 25: 1960-67. [Crossref]

17. Kasper S, Volz H, Dienel A, Schlafke S (2016) Efficacy of Silexan in mixed anxietydepression - A randomized, placebo-controlled trial. Euro Neuropsychopharmacol 26 331-340. [Crossref]

18. Generoso MB, Soares A, Taiar IT, Cordeiro Q, Shiozawa P (2017) Lavender Oil Preparation (Silexan) for Treating Anxiety: An Updated Meta-Analysis. J Clin Psychopharmacol 37: 115-117. [Crossref]

19. Kasper S (2013) An orally administered lavandula oil preparation (Silexan) for anxiety disorder and related conditions: an evidence based review. Int J Psychiatry Clin Pract 17: 15-22. [Crossref]

20. Food and Drug Administration (2016) Department of Health and Human Services. Substances Generally Recognized as Safe. Code of Federal Regulations.

21. Baldinger P, Höflich AS, Mitterhauser M, Hahn A, Rami C, et al. (2015) Effects of Silexan on the serotonin-1A receptor and microstructure of the human brain: a randomized, placebo-controlled, double-blind, cross-over study with molecular and structural neuroimaging. Int J Neuropsychopharmacol 18: 63. [Crossref]

22. Bose J, Hedden S, Lipari R, Park-Lee E, Porter J, et al. (2015) Key Substance Use and Mental Health Indicators in the United States: Results from the 2015 National Survey on Drug Use and Health.

23. P. Peggy Hsu (2002) Natural Medicines Comprehensive Database. J Med Libr Assoc 90: 114 [Crossref]

Copyright: (C2018 Henneman A. This is an open-access article distributed under the terms of the Creative Commons Attribution License, which permits unrestricted use, distribution, and reproduction in any medium, provided the original author and source are credited. 